\title{
GENERALIZED PROJECTORS AND CARTER SUBGROUPS OF FINITE GROUPS
}

\author{
SURINDER K. SEHGAL
}

\begin{abstract}
Projectors of finite, solvable groups were defined by Schunk [6]. In this paper, the author defines generalized projectors of finite groups. They are the same as projectors in the case of solvable groups. The existence of this class of subgroups is shown for $\pi$-solvable groups satisfying certain properties.
\end{abstract}

Let $\chi$ be a class of groups, which is closed under isomorphisms and $1 \in \chi$.

DEFINITION. $S$ is $\chi$-maximal in $G$ if (i) $S$ is a subgroup of $G$, (ii) $S \in \chi$, (iii) $S \subseteq T \subseteq G \& T \in \chi \Rightarrow S=T$.

DEFINITION. $S$ is a $\chi$-projector of $G$ if (i) $S$ is a subgroup of $G$, (ii) $H \unlhd G \Rightarrow H S / H$ is $\chi$-max in $G / H$.

DEFINITION. $\chi$ is called a Schunk class if $\chi$ satisfies the following conditions: (a) if $G \in \chi, H \unlhd G$ then $G / H \in \chi$, (b) whenever all primitive factor groups of a group $G$ are in $\chi$ then so is $G$ in $\chi$.

Schunk [6] proved the following theorem.

THEOREM. If $\chi$ is a Schunk class then every finite, solvable group $G$ has $a \chi$-projector and all $\chi$-projectors are conjugate.

Definition. Let $S$ be a class of subgroups of a finite group $G$. Then we say $S$ is a characteristic class of conjugate subgroups if (i) $H \in S$ and $\alpha$ an automorphism of $G$ then $H^{\alpha} \in S$, (ii) $H^{\alpha}$ is a conjugate of $H$ under an element of $G$ i.e., $\exists g \in G$ such that $H^{\alpha}=H^{g}$.

Definition. A finite group $G$ is called $\pi$-closed if it contains a normal subgroup $N$ which is a $\pi$-group and $G / N$ is a $\pi^{\prime}$-group.

All groups considered in this paper are finite.

Let $\chi$ be any Schunk class consisting of solvable, $\pi$-closed groups. In this paper we will show that if the $\pi^{\prime}$-subgroups of a $\pi$-solvable group $G$ have $\chi$-projectors and they form a characteristic class of conjugate subgroups then $G$ has $\chi$-projectors and the $\chi$-projectors form a characteristic class of conjugate subgroups. We cannot expect that all $\chi$-projectors are conjugate as is clear from the case when $G$ is a finite, nonabelian simple

Received by the editors October 18, 1971 and, in revised form, April 26, 1972.

AMS (MOS) subject classifications (1969). Primary 2054.

(c) American Mathematical Society 1973 
group and $\chi=\mathfrak{N}$ the class of all nilpotent groups. As can be easily seen, the generalized Carter subgroups as defined in $\S 2$ are $\mathfrak{N}$-projectors, but the converse is not true. We are unable to prove that $\chi$-projectors exist and they form a characteristic class of conjugate subgroups in the case when $G / N \in \chi$ and $N$ is a direct product of isomorphic copies of simple nonabelian groups. In case the existence of $\chi$-projectors can be shown in this case then proofs similar to the ones given below prove that $\chi$-projectors exist in all finite groups and they form a characteristic class of conjugate subgroups.

1. Definition. The descending Loewy series is defined as the characteristic chain

$$
G=\Lambda_{0}(G) \supset \Lambda_{1}(G) \supset \cdots \supset \Lambda_{k}(G)=\{1\}
$$

where $\Lambda_{1}(G)=\Lambda(G)$ is the intersection of all the maximal normal subgroups of $G$ and $\Lambda_{i+1}(G)=\Lambda_{1}\left(\Lambda_{i}(G)\right)$.

Denote by $k$ the length of the descending Loewy series.

REMARK. $\Lambda(G)=\{1\}$ if and only if $G$ is a direct product of simple groups.

Definition. A group $G$ is called semisimple if $G$ is isomorphic to a direct product of simple groups.

THEOREM 1.1. If $\alpha \in \operatorname{Hom}(G)$ then $\Lambda_{i}\left(G^{\alpha}\right)=\Lambda_{i}(G)^{\alpha}$.

Proof. Let $\subseteq$ be the set of all finite semisimple groups. Then $\subseteq$ is a

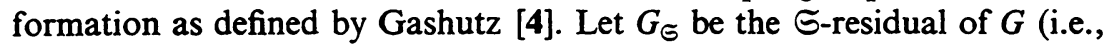
the smallest normal subgroup of $G$ satisfying $G / G_{\Im} \in \Xi$ ). Obviously $G_{G}=\Lambda_{1}(G)$. Now by a lemma of Gashutz [4], the result follows.

Definition. A subgroup $U$ of a finite group $G$ is called a generalized $\chi$-projector of $G$, if it satisfies the following conditions for all homomorphisms $f$ of $G$.

(1) $f(U)$ is a $\chi$-max subgroup of $f(G)$.

(2) $\{f(U)\}$ is a characteristic class of conjugate subgroups of $f(G)$.

REMARK. It is clear that generalized $\chi$-projectors are the same as $\chi$ projectors in the case when $G$ is solvable.

REMARK. If $U$ is a generalized $\chi$-projector of $G, N \unlhd G$ then $U N / N$ is a generalized $\chi$-projector of $G / N$.

REMARK. The class of generalized $\chi$-projectors of $G$ forms a characteristic class of conjugate subgroups.

LEMMA 1.2. Let $N$ be a normal subgroup of $G$ such that $N^{\sigma}$ is a characteristic subgroup of $G^{\sigma}$ for each homomorphism $\sigma$ of $G$. Let $U / N$ be a generalized $\chi$-projector of $G / N$ and $U$ a generalized $\chi$-projector of $U$; then $U$ is $a \chi$ projector of $G$. 
Proof. It is enough to show that $U M / M$ satisfies conditions (i) and (ii) in $G / M$ where $M$ is any normal subgroup of $G$. Suppose $U M / M \subseteq V \mid M$; $V / M \in \chi$. Since $U N M / N M$ is a generalized $\chi$-projector of $G / N M ; V N / N M$ $\in \chi$, therefore $V N / N M=U N M / N M$ or $V / M \subseteq U M / M$ but then $U M / M$ is a generalized $\chi$-projector of $U / M$ hence $U M / M=V \mid M$. For (ii) let $\alpha$ be any automorphism of $G / M$. Then $\alpha$ induces an automorphism $\bar{\alpha}$ of $G / N M$, but $U N M / N M$ is a generalized $\chi$-projector of $G / N M$. Therefore $(U N M / N M)^{\bar{\alpha}}=(U N M / N M)^{g}$ for some $g \in G$ or $(U M / M)^{\alpha g^{-1}}=U M / M$ but $U M / M$ is a generalized $\chi$-projector of $U / M$ so $(U M / M)^{\alpha}$ is a conjugate of $U M / M$. Hence $U$ is a generalized $\chi$-projector of $G$.

REMARK. A homogeneous component of $\Lambda_{k-1}(G)$ has the property described in Lemma 1.2.

THEOREM 1.3. Let $\pi$ be a set of primes and $\chi$ a Schunk class consisting of solvable $\pi$-closed groups. If the $\pi^{\prime}$-subgroups of a $\pi$-solvable group $G$ have generalized $\chi$-projectors then so does $G$.

Proof. Suppose the result is not true. Let $G$ be a group of smallest possible order for which the result is false. Suppose there exists a nontrivial solvable $\pi$-subgroup $N$ of $G$ satisfying $N^{\sigma}$ which is a characteristic subgroup of $G^{\sigma}$ for $\sigma$ any homomorphism of $G$. Then using the SchurZassenhaus complement theorem [7], $G / N$ is not a criminal and so has a generalized $\chi$-projector say $\boldsymbol{U} / N$. Now $\boldsymbol{U}$ is solvable, therefore has a generalized $\chi$-projector say $U$. By Lemma $1.2, U$ is a generalized $\chi$ projector of $G$ which cannot happen. Now let $N$ be a homogeneous component of $\Lambda_{k-1}(G)$. So we can assume $N$ is a $\pi^{\prime}$-group. $G / N$, being not a criminal, has a generalized $\chi$-projector say $\boldsymbol{U} / N$. If $\boldsymbol{U}$ is a proper subgroup of $G$ then $U$ has a generalized $\chi$-projector say $U$ and, by Lemma 1.2, $U$ is a generalized $\chi$-projector of $G$. So $U=G$. Now $G$ cannot be a $\pi^{\prime}$-group by hypothesis and it is clearly true that $G$ has Sylow $\pi$-subgroups and all Sylow $\pi$-subgroups of $G$ are conjugate. Let $P$ be a Sylow $\pi$-subgroup of $G$. Since $P$ is solvable, $P$ cannot be normal in $G$. So $N_{G}(P)$ is a proper subgroup of $G$. Let $U$ be a generalized $\chi$-projector of $N_{G}(P)$. We will show that $U$ is a generalized $\chi$-projector of $G$. Since $G / N \in \chi$, a Sylow $\pi$-subgroup of $G / N$ is normal in $G / N$. So $N_{G}(P) N / N=N_{G / N}(P N / N)=G / N$. Now $U N / N$ is a generalized $\chi$-projector of $N_{G}(P) N / N=G / N$ and $G / N \in \chi \Rightarrow U N / N=G / N$ so a Sylow $\pi$-subgroup of $U$ is a Sylow $\pi$-subgroup of $G$ but $U \subseteq N_{G}(P) \Rightarrow P$ is a Sylow $\pi$-subgroup of $U$ and $P \unlhd U$. Let $M$ be any normal subgroup of $G$. We will now show that $U M / M$ satisfies conditions (i) and (ii). Suppose $U M|M \subseteq V| M ; V / M \in \chi$. So a Sylow $\pi$-subgroup of $V / M$ is $P M / M$ and, by the choice of $\chi, P M|M \unlhd V| M$ or $V \mid M \subseteq N_{G / M}(P M / M)=N_{G}(P) \cdot M / M$ 
but $U M / M$ is a generalized $\chi$-projector of $N_{G}(P) M / M$. Hence $U M / M=$ $V \mid M$. For (ii) let $\alpha$ be any automorphism of $G / M$. Then $\left(N_{G}(P) M / M\right)^{\alpha}=$ $\left(N_{G / M}(P M / M)\right)^{\alpha}=N_{G / M}(P M / M)^{g}$ or $\left(N_{G}(P) M / M\right)^{\alpha g-1}=N_{G}(P) M / M$, but $U M / M$ is a generalized $\chi$-projector of $N_{G}(P) M / M$. Hence $(U M / M)^{\alpha}$ is a conjugate of $U M / M$. So $U$ is a generalized $\chi$-projector of $G$.

2. Ja. G. Berkovič [1] has shown that if the $\pi^{\prime}$-subgroups of a $\pi$-solvable group $G$ have a Carter subgroup and all the Carter subgroups are conjugate there then $G$ also has a Carter subgroup and all Carter subgroups are conjugate. In this section we will show that if the $\pi^{\prime}$-subgroups of a $\pi$ solvable group $G$ have a generalized Carter subgroup and they form a characteristic class of conjugate subgroups there then $G$ has a generalized Carter subgroup and the generalized Carter subgroups form a characteristic class of conjugate subgroups.

Definition. A subgroup $U$ of a finite group $G$ is called a generalized Carter subgroup of $G$ if $U$ satisfies the following conditions:

(a) $U$ is nilpotent.

(b) $N_{G}(U)=U$.

(c) If $\alpha$ is any automorphism of $G$ then $U^{\alpha}$ is a conjugate of $U$ under an element of $G$ i.e., $\exists g \in G$ such that $U^{\alpha}=U^{g}$.

(d) $U N / N$ satisfies conditions (a), (b), and (c) in $G / N$ where $N$ is any normal subgroup of $G$.

REMARK. If $G$ is solvable then generalized Carter subgroups are the same as the Carter subgroups of solvable groups.

REMARK. If the $\pi^{\prime}$-subgroups of a $\pi$-solvable group $G$ have a generalized Carter subgroup then the class of generalized Carter subgroups of $G$ forms a characteristic class of conjugate subgroups.

REMARK. If the $\pi^{\prime}$-subgroups of a $\pi$-solvable group $G$ have a generalized Carter subgroup then the homomorphic image of a generalized Carter subgroup $U$ under a group homomorphism $\sigma$ of $G$ is a generalized Carter subgroup of $G^{\sigma}$.

LEMma 2.1. Let $N$ be a normal subgroup of $G$ such that $N^{\sigma}$ is a characteristic subgroup of $G^{\sigma}$ for each homomorphism $\sigma$ of $G$. Let $U / N$ be a generalized Carter subgroup of $G / N$ and $U$ a generalized Carter subgroup of $G$. Then $U$ is a generalized Carter subgroup of $G$.

Proof. Similar to the proof of Lemma 1.2.

THEOREM 2.2. If the $\pi^{\prime}$-subgroups of a $\pi$-solvable group $G$ have generalized Carter subgroups, then so does $G$.

Proof. Suppose the result is false. Let $G$ be a group of smallest possible order for which the result is not true. Let $N$ be a homogeneous component 
of $\Lambda_{k-1}(G)$. Then $G / N$ is not a criminal and so has a generalized Carter subgroup say $\boldsymbol{U} / N$. If $\boldsymbol{U}$ is a proper subgroup of $G$ then $\boldsymbol{U}$ has a generalized Carter subgroup, say $U$, and by Lemma 2.1, $U$ is a generalized Carter subgroup of $G$. Hence we can assume $\bar{U}=G, N$ is a $\pi^{\prime}$-group and $G$ is not a $\pi^{\prime}$-group. Let $p$ be a prime in $\pi$ such that $p$ divides the order of $G$. Let $P$ be a Sylow $p$-subgroup of $G$. If $P$ is normal in $G$ then $G=P \times G_{1},\left|G_{1}\right|<|G| \Rightarrow G_{1}$ has a generalized Carter subgroup, say $U_{1}$. Then, as can be easily seen, $U=S_{p} \times U_{1}$ is a generalized Carter subgroup of $G$. So we can assume that $N_{G}\left(S_{P}\right)$ is a proper subgroup of $G$. Let $U$ be a generalized Carter subgroup of $N_{G}\left(S_{P}\right)$. We claim that $U$ is a generalized Carter subgroup of $G$. Let $M$ be any normal subgroup of $G$. It is enough to show $U M / M$ satisfies conditions (a), (b) and (c) in $G / M$. (a) Clearly $U M / M$ is nilpotent. (b) $N_{G / M}(U M / M) \subseteq N_{G / M}\left(S_{p} M / M\right)=N_{G}\left(S_{p}\right) M / M \Rightarrow N_{G / M}(U M / M)=$ $U M / M$. (c) If $\propto$ is any automorphism of $G / M$, then $U M / M \subseteq N_{G}\left(S_{p}\right) M / M=$ $N_{G / M}\left(S_{p} M / M\right)$. Now $N_{G / M}\left(S_{p} M / M\right)^{\alpha}=N_{G / M}\left(S_{p} M / M\right)^{g}$ for some $g \in$ $G / M$ or $\left(N_{G}\left(S_{p}\right) M / M\right)^{\alpha g^{-1}}=N_{G}\left(S_{p}\right) M / M$. But $U$ is a generalized Carter subgroup of $N_{G}\left(S_{p}\right)$. Therefore $(U M / M)^{\alpha g-1}$ is a conjugate of $U M / M$ or $(U M / M)^{\alpha}$ is a conjugate of $U M / M$. Hence $U$ is a generalized Carter subgroup of $G$.

\section{REFERENCES}

1. Ja. G. Berkovič, Generalization of the theorems of Carter and Wielandt, Dokl. Akad. Nauk SSSR 171 (1966), 770-773=Soviet Math. Dokl. 7 (1966), 1525-1529. MR 34 \#7647.

2. R. W. Carter, On a class of finite soluble groups, Proc. London Math. Soc. (3) 9 (1959), 623-640. MR 22 \#5677.

3. - Nilpotent self-normalizing subgroups and system normalizers, Proc. London Math. Soc. (3) 12 (1962), 535-563. MR 25 \#3988.

4. W. Gashütz, Zur theorie der endlichen auflösbaren Gruppen, Math. Z. 80 (1962/63), 300-305. MR 31 \#3505.

5. B. Huppert, Endliche Gruppen. I, Die Grundlehren der math. Wissenschaften, Band 134, Springer-Verlag, Berlin and New York, 1967. MR 37 \#302.

6. H. Schunk, $\mathfrak{-}$-untergruppen in endlichen auflösbaren Gruppen, Math. Z. 97 (1967), 326-330 MR 35 \#254.

7. H. Zassenhaus, Lehrbuch der Gruppentheorie, Teubner, Leipzig, 1937; English transl., Chelsea, New York, 1949. MR 11, 77.

Department of Mathematics, Ohio State University, Columbus, Ohio 43210 